\title{
Atwood number effects on the instability of a uniform interface driven by a perturbed shock wave
}

\author{
Shenfei Liao, ${ }^{1, *}$ Wenbin Zhang, ${ }^{1}$ Hu Chen, ${ }^{1, \dagger}$ Liyong Zou, ${ }^{1,2}$ Jinhong Liu, ${ }^{1}$ and Xianxu Zheng ${ }^{1}$ \\ ${ }^{1}$ Laboratory for Shock Wave and Detonation Physics, Institute of Fluid Physics, China Academy of Engineering Physics, \\ P.O. Box 919-103, Mianyang, Sichuan 621900, China \\ ${ }^{2}$ Mechanical Engineering, Physical Science and Engineering Division, King Abdullah University of Science and Technology, \\ Thuwal 23955-6900, Saudi Arabia
}

(Received 25 July 2018; published 8 January 2019)

\begin{abstract}
The evolution of a uniform interface subjected to a perturbed shock wave has been experimentally studied over a range of Atwood numbers $0.22 \leqslant A \leqslant 0.68$ and Mach numbers $1.2 \leqslant M \leqslant 1.8$ using a vertical shock tube. The perturbed shock wave is produced by diffracting a planar incident shock over a rigid cylinder. The wave patterns of the perturbed shock are captured by high-speed shadowgraphy, while the evolution of the shocked interface is captured by planar Mie scattering. Besides the formations of a cavity and two steps, an apparent counter-rotating vortex pair emerges on the shocked interface due to the baroclinic vorticity deposition, as both the Atwood number and Mach number increase. Quantitatively, it is interesting to note that the amplitude growth rate of the shocked interface decreases with increasing the Atwood number, which is fundamentally different from the results related to the classical RM instability. This notable feature is explained by the approximation of an oblique shock hitting a uniform interface. For weak shock, a suitable time scaling is employed to collapse experimental data irrespective of the Atwood number difference.
\end{abstract}

DOI: 10.1103/PhysRevE.99.013103

\section{INTRODUCTION}

The classical Richtmyer-Meshkov (RM) instability [1,2], arising when a uniform (i.e., without perturbation) shock wave passes through an initially perturbed interface between two different fluids, has attracted significant attention because of its fundamental importance not only for natural phenomena such as supernova explosions [3], but also for practical applications such as inertial confinement fusion [4]. As it has been comprehensively reviewed by Zabusky [5], Brouillette [6], Ranjan et al. [7], and most recently by Zhou [8,9] and Zhai et al. [10], significant progress has been made in the study of the classical RM instability and the instability-induced mixing.

Note that in the classical RM instability the shock wave is idealized as being uniform, and only the perturbation on the interface is taken into account. However, the shock wave that drives the interface instability is usually disturbed before it impacts the interface in reality. For example, in type II supernova, the radially expanding shock wave is distorted when it crosses from the collapsed core into the overlaying layers because of asphericities in the core, convection behind the shock during shock formation, and density perturbations in the layers [11]. In inertial confinement fusion, the shock wave that drives target implosion is essentially perturbed because it generates from nonuniform ablation caused by nonuniform laser irradiation. Thus, the study of the interface

\footnotetext{
*sfliao@caep.cn

${ }^{\dagger}$ Present address: Automotive Engineering Institute, Guangzhou Automobile Group Co., LTD, Guangzhou, Guangdong 511434, China.
}

instability driven by perturbed shock waves is essential for a better understanding of the complex hydrodynamic instability in reality.

Compared to the classical RM instability, the interface instability driven by a perturbed shock wave has been little studied in the literature. Ishizaki et al. [12] numerically investigated the instability of a uniform interface accelerated by a nonuniform shock wave, which was generated by a rippled rigid piston moving with constant velocity. They found that the uniform interface became unstable after being impacted by the nonuniform shock, and the perturbation growth rate depended sensitively on the phase of the oscillating shock at the impinging moment. Furthermore, they observed evolution differences between this instability and the classical RM instability, which, however, has not been confirmed by experiments so far. Zhang et al. [13] also performed a numerical study on this instability, where a sinusoidal rippled shock was generated by a planar shock passing through a single-mode interface. They found that the influence of the shock strength on the instability growth was different for rippled shocks at different phases. On the Omega laser system, Kane et al. [11] reported the first experimental observation showing this nonuniform shock induced instability. The nonuniform shock was generated by a planar incident shock passing through a rippled interface. From the observation of instability growth with opposite phases at the initially single-mode interface and initially uniform interface, they stated that the perturbation growth on the uniform interface was indeed seeded by the nonuniform shock. Recently, Zou et al. [14] performed a novel shock-tube experiment, where a perturbed shock wave was generated by a planar incident shock diffracting over a rigid cylinder. They found that the growth rate of the instability predicted by Richtmyer's impulsive model was much 
larger than the experimental counterpart, provided that the pre-interface amplitude of the perturbed shock was equated to the pre-shock amplitude of a perturbed interface. Similar shock-tube experiments were carried out with multiple rigid cylinders in converging geometry by Liang et al. [15], and in planar geometry by Zhai et al. [16]. They confirmed that the interface evolution was also influenced by cylinder number and spacing between cylinders. In addition, a modified impulsive model was proposed and could reasonably predict the perturbation growth rate by taking the experimentally measured post-shock perturbation of the interface as an initial interface perturbation. Note that only a single Atwood number, indicating density contrast at the interface, is considered in all the experiments mentioned above, and thus the effect of the Atwood number has not yet been explored. However, it has been demonstrated in the classical RM instability that the Atwood number has a significant influence on both the linear growth rate of the perturbation $[1,2]$ and the late-time mixing efficiency [17]. Furthermore, due to the differences in physical mechanism and the lack of experimental data, whether Richtmyer's impulsive model proposed for the classical RM instability is applicable to the interface instability driven by a perturbed shock wave remains an open issue.

In this work, the instability of a uniform interface driven by a perturbed shock wave is experimentally studied. The perturbed shock is generated by the diffraction of a planar incident shock over a rigid cylinder. The uniform interfaces with three different initial Atwood numbers are formed membranelessly. The wave patterns of the perturbed shock and the interface evolution are quantitatively characterized by highspeed shadowgraphy and planar Mie scattering, respectively. The Atwood number effects on the perturbation growth of the uniform interface are quantitatively analyzed.

\section{EXPERIMENTAL METHOD}

The experiments were conducted in a similar vertical shock tube with a 100-mm-square cross section as in our previous work [14]. The shock tube has a 1.60-m-long driver section, a 4.22-m-long driven section, and a $0.305-\mathrm{m}$-long test section. A polyester diaphragm separating the driver and driven sections is ruptured by heating thin metal wires in contact with the diaphragm through a powerful electrical pulse, which achieves a controllable and highly reproducible planar incident shock. Three cases of planar incident shocks with Mach numbers of $M=1.20 \pm 0.01,1.50 \pm 0.01$, and $1.80 \pm 0.01$ were produced. After the incident shock diffracts over a rigid circular cylinder in the test section, a perturbed shock is generated and then propagates downstream, until it finally impacts a uniform interface, as illustrated in Fig. 1. The diameter of the cylinder, $d$, is $10 \mathrm{~mm}$, while the distance between the cylinder center and the interface, $l$, is $40 \mathrm{~mm}$, as schematically shown in Fig. 1(b). Both $d$ and $l$ are kept constant in the present study.

A uniform interface was created using the membraneless method proposed by Jones and Jacobs [18]. Therefore, there is no membrane effect that may influence the evolution of the shocked interface. The light gas is flowed downwards from the top of the driven section while the heavy gas is flowed upwards from the bottom of the test section. Both gases meet
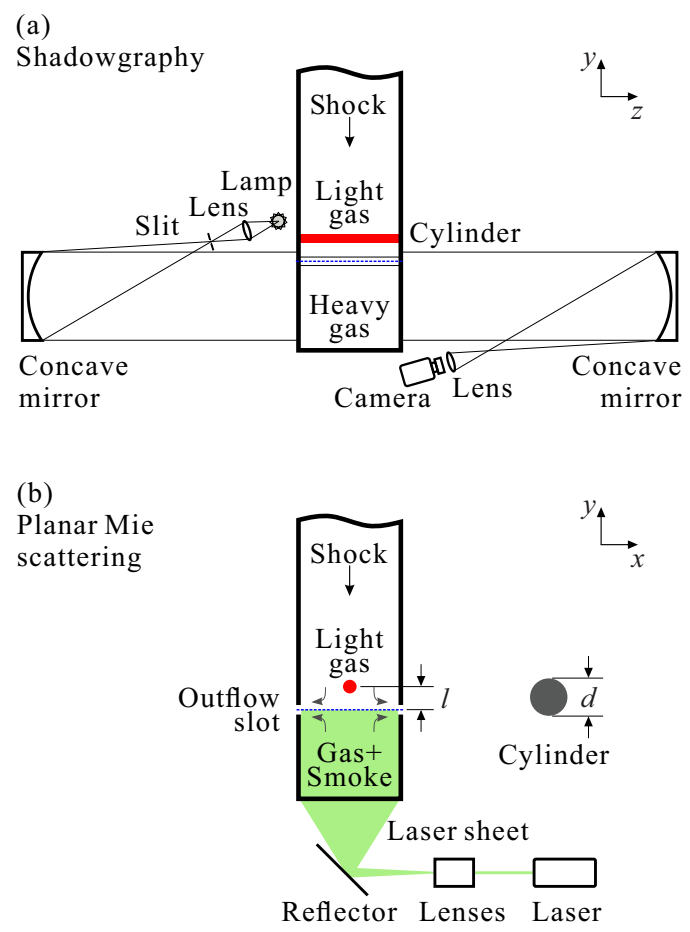

FIG. 1. Schematics of the test section of the shock tube with two visualization techniques: (a) shadowgraphy and (b) planar Mie scattering.

at the outflow slots in the test section walls and exit, resulting in a nominally flat, uniform interface, as schematically shown in Fig. 1(b). To investigate the Atwood number effects, three different types of fast-slow interfaces were formed with light gas of nitrogen $\left(\mathrm{N}_{2}\right)$ and heavy gases of carbon dioxide $\left(\mathrm{CO}_{2}\right)$, krypton $(\mathrm{Kr})$, and sulfur hexafluoride $\left(\mathrm{SF}_{6}\right)$, respectively. Accordingly, the initial Atwood numbers of the interfaces are $A=0.22,0.50$, and 0.68 , where $A$ is defined as $A=$ $\left(\rho_{2}-\rho_{1}\right) /\left(\rho_{2}+\rho_{1}\right)$ with $\rho_{1}\left(\rho_{2}\right)$ being the density of the light (heavy) fluid. The width of the two outflow slots is $6 \mathrm{~mm}$, which is approximate to that in Ref. [18]. As a result, the flow rates of the light and heavy gases are taken to be $1.12 \mathrm{~m}^{3} / \mathrm{h}$ and $0.36 \mathrm{~m}^{3} / \mathrm{h}$, respectively, which are similar to those yielding the thinnest interface with least diffusion, as recommended by Jones and Jacobs [18].

Shadowgraphy and planar Mie scattering were employed to capture the wave patterns of the perturbed shock and the interface evolution, respectively. For the shadowgraphy, as illustrated in Fig. 1(a), the flow field is illuminated by a 500-W xenon lamp (XQW500, Chengdu Photoelectricity Limited) and exhibited by a high-speed video camera (Phantom V1610) with a frame rate of $5.1 \times 10^{5} \mathrm{fps}$ and an exposure time of $1 \mu \mathrm{s}$. The planar Mie scattering is used to quantitatively characterize the evolution of the shocked interface, as shown in Fig. 1(b). The heavy test gas is seeded with filtered cigarette smoke, which was widely used as tracer particles because of its small and roughly similar particle size (less than $0.5 \mu \mathrm{m})$ in previous shock-tube experiments [14,19-21] with Mach numbers ranging from 1.13 to 3.08. After the uniform interface is formed with sufficient gas purity on each side, cigarette smoke is inserted into the flow of the heavy gas. 

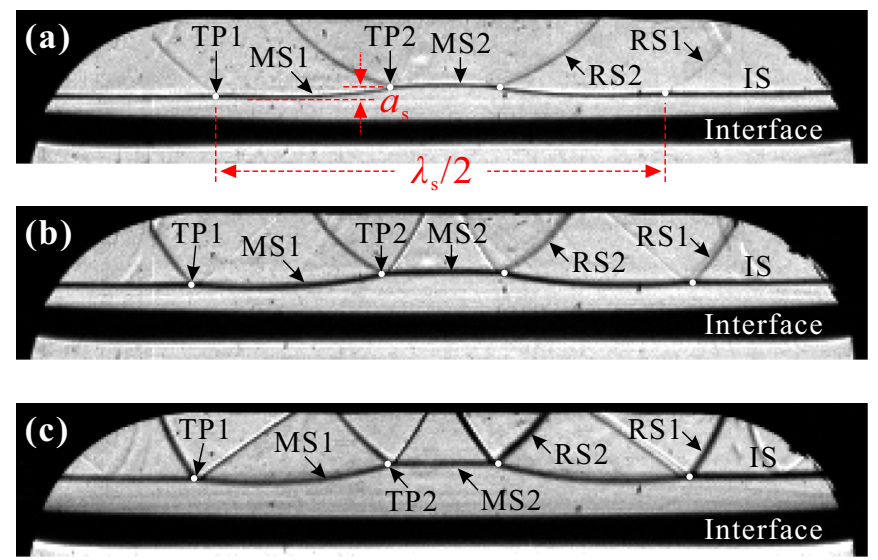

FIG. 2. Shadowgraph pictures showing the wave patterns of the perturbed shock just before the shock impacts the uniform interface: (a) $M=1.2$; (b) $M=1.5$; (c) $M=1.8$. The perturbed shock propagates from top to bottom with amplitude $a_{\mathrm{s}}$ and wavelength $\lambda_{\mathrm{s}}$. Notation: IS, incident shock; RS, reflected shock; MS, Mach stem; $\mathrm{TP}$, triple point.

Before firing the shock wave, suitable time is controlled for obtaining a uniform gas-smoke mixture and having sufficient amount of smoke to realize a high-quality image. The Mie scattering light source is a Nd:YAG $532 \mathrm{~nm}$ laser (SGR-Extra, Beamtech Limited), which can produce 30 pulses with each pulse duration of $10 \mathrm{~ns}$ and energy of $\sim 100 \mathrm{~mJ}$ at a frequency of $10 \mathrm{kHz}$. A laser sheet (thickness of $\sim 1 \mathrm{~mm}$ ), produced by the laser pulse passing through circular and cylindrical lenses, is reflected upward through the glass window fixed at the lower end of the shock tube and illuminates the flow field. Mie scattering sequences, showing the evolutions of the shocked interfaces, are then captured by a camera aligned normal to the light sheet. The frame rate and exposure time of the camera are $1.0 \times 10^{4} \mathrm{fps}$ and $99 \mu \mathrm{s}$, respectively.

\section{RESULTS AND DISCUSSION}

\section{A. Wave patterns}

Diffraction of a planar shock over a circular cylinder is a classical shock dynamics problem [22,23], which includes regular reflection, transition to Mach reflection and complex shock-shock interaction. Due to the symmetry of the diffraction system, a vertical symmetry plane parallel with the $y-z$ plane shown in Fig. 1 can be considered through the center of the cylinder. On either side of the symmetry plane, the diffracted perturbed shock, right before it impacts the uniform interface, consists of an incident shock (IS), two Mach stems (MS1, MS2), and two reflected shocks (RS1, RS2), as shown in Fig. 2. The MS1 is separated from the IS and the MS2 by the two triple points TP1 and TP2, respectively. The shock strength experiences a sudden jump upon crossing the TP1 from the IS to the MS1, then decreases continuously along the MS1, and reaches a local minimum value at the TP2 [23]. Similarly, there is a sudden jump in the shock strength upon crossing the TP2 from the MS1 to the MS2 [24].

The wave patterns of the perturbed shock for the three Mach numbers are qualitatively similar, as shown in Fig. 2. However, distinguishable differences can still be observed that

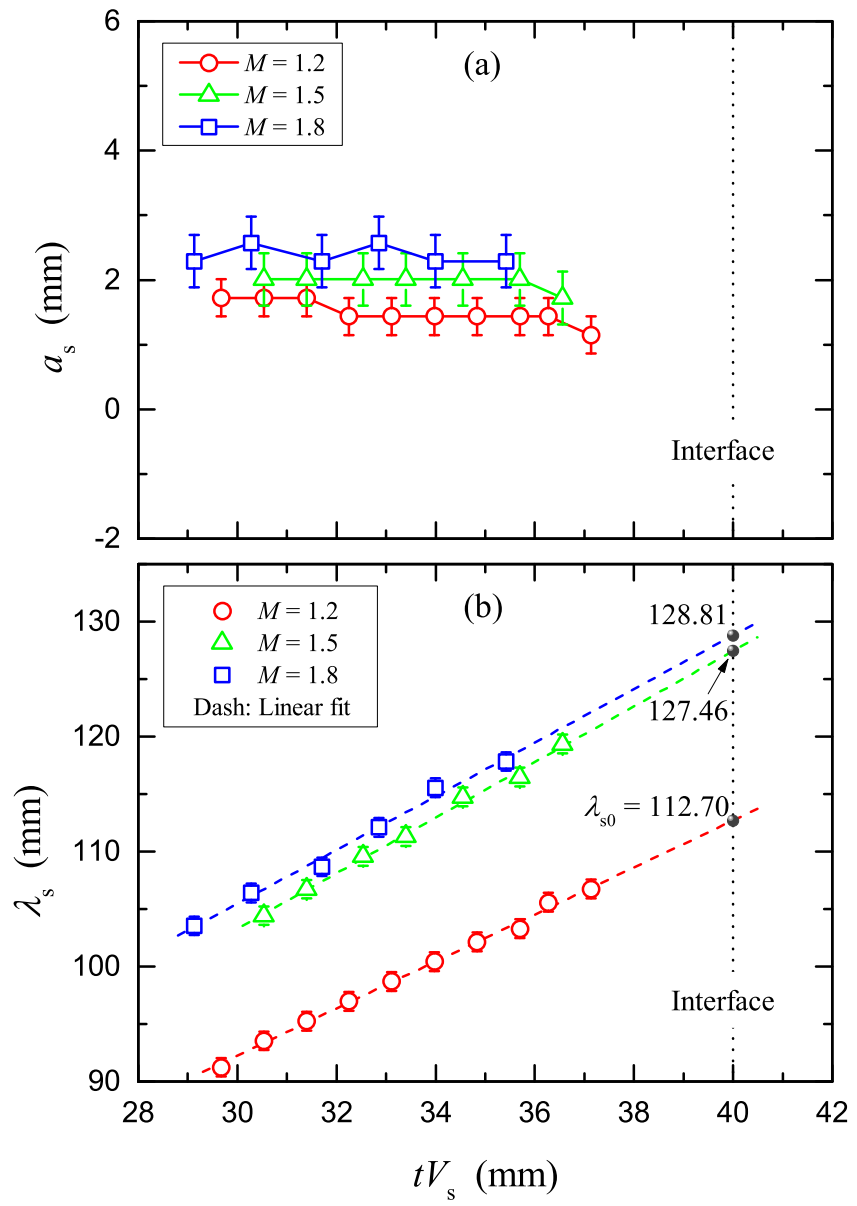

FIG. 3. Variations of the amplitude $a_{\mathrm{s}}$ (a) and the wavelength $\lambda_{\mathrm{s}}$ (b) of the perturbed shock with the distance between the incident shock (IS) and the cylinder center, $t V_{\mathrm{s}}$. The dotted line indicates the location of the uniform interface.

as the Mach number increases the MS1 becomes more curved while the MS2 becomes more planar. Due to the relatively low frame rate of high-speed video camera (i.e., $6 \times 10^{3} \mathrm{fps}$ ), only one schlieren picture of the perturbed shock can be obtained in each shot before the perturbed shock impacts the interface in our previous work [14]. However, the marked improvement in the frame rate in the present study (i.e., $5.1 \times 10^{5} \mathrm{fps}$ ) enables us to record time sequences of the perturbed shock propagation, and thus makes it possible to provide more accurate measurements of the perturbed shock. More quantitative measurements of the perturbed shock are shown in Fig. 3, where the amplitude $a_{\mathrm{s}}$ and the wavelength $\lambda_{\mathrm{s}}$ are defined in the same way as a perturbed single-mode interface in the classical RM instability, as illustrated in Fig. 2(a). The abscissa, $t V_{\mathrm{s}}$, is the distance between the IS and the cylinder center, where $V_{\mathrm{s}}$ is the velocity of the IS and the initial time, $t=0$, is defined as the time the IS passes across the cylinder center. The error bars represent the experimental uncertainty. Due to the limitation of the camera, it is difficult to exactly capture the amplitude $a_{\mathrm{s} 0}$ and wavelength $\lambda_{\mathrm{s} 0}$ corresponding to the time when the perturbed shock impacts the interface. Approximations of $a_{\mathrm{s} 0}$ and $\lambda_{\mathrm{s} 0}$ are taken in the present work. As shown in Fig. 3(a), the amplitude of the perturbed shock increases with increasing 
TABLE I. Comparison of the amplitude growth rate as given by Richtmyer's impulsive model and experiments. $a_{\mathrm{s} 0}$ and $\lambda_{\mathrm{s} 0}$ are the amplitude and wavelength of the perturbed shock, respectively, corresponding to the time when the perturbed shock impacts the uniform interface. $A$ and $A^{+}$are the pre-shock and post-shock Atwood numbers, respectively. $a_{\mathrm{i} 0}$ is the post-shock initial amplitude of the interface estimated by Eq. (2). $\lambda_{\mathrm{i} 0}$ is the post-shock initial wavelength of the interface measured from Mie scattering pictures. $\Delta V$ is the velocity jump of the shocked interface calculated based on the one-dimensional gas dynamics and the discontinuous interface approximation. $\dot{a}_{\text {eq } 1}$ and $\dot{a}_{\text {eq } 2}$ are the amplitude growth rates predicted by Richtmyer's impulsive model with initial amplitudes of $a_{\mathrm{s} 0}$ and $a_{\mathrm{i} 0}$, respectively. $\dot{a}_{\mathrm{exp}}$ is the amplitude growth rate calculated from the experimental results.

\begin{tabular}{|c|c|c|c|c|c|c|c|c|c|c|c|}
\hline$M$ & $a_{\mathrm{s} 0}(\mathrm{~mm})$ & $\lambda_{\mathrm{s} 0}(\mathrm{~mm})$ & $a_{\mathrm{s} 0} / \lambda_{\mathrm{s} 0}$ & $A$ & $A^{+}$ & $a_{\mathrm{i} 0}(\mathrm{~mm})$ & $\lambda_{\mathrm{i} 0}(\mathrm{~mm})$ & $\Delta V(\mathrm{~m} / \mathrm{s})$ & $\dot{a}_{\mathrm{eq} 1}(\mathrm{~m} / \mathrm{s})$ & $\dot{a}_{\mathrm{eq} 2}(\mathrm{~m} / \mathrm{s})$ & $\dot{a}_{\exp }(\mathrm{m} / \mathrm{s})$ \\
\hline \multirow[t]{3}{*}{1.2} & 1.15 & 112.70 & 0.01 & 0.22 & 0.23 & 0.26 & 117.01 & 99.23 & 1.46 & 0.32 & 1.87 \\
\hline & & & & 0.50 & 0.47 & 0.20 & 116.72 & 78.82 & 2.38 & 0.40 & 1.45 \\
\hline & & & & 0.68 & 0.71 & 0.18 & 114.40 & 70.77 & 3.22 & 0.50 & 1.34 \\
\hline \multirow[t]{3}{*}{1.5} & 1.72 & 127.46 & 0.01 & 0.22 & 0.25 & 0.70 & 128.59 & 218.57 & 4.63 & 1.87 & 10.86 \\
\hline & & & & 0.50 & 0.44 & 0.56 & 129.17 & 176.03 & 6.57 & 2.11 & 8.68 \\
\hline & & & & 0.68 & 0.74 & 0.51 & 128.88 & 158.87 & 9.97 & 2.92 & 5.07 \\
\hline \multirow[t]{3}{*}{1.8} & 2.29 & 128.81 & 0.02 & 0.22 & 0.26 & 1.15 & 129.92 & 327.21 & 9.50 & 4.73 & 24.06 \\
\hline & & & & 0.50 & 0.40 & 0.93 & 129.37 & 263.91 & 11.79 & 4.77 & 22.88 \\
\hline & & & & 0.68 & 0.76 & 0.84 & 130.54 & 239.12 & 20.30 & 7.35 & 12.73 \\
\hline
\end{tabular}

the Mach number. Generally, the amplitude for each Mach number changes little with the distance, though it tends to decrease with the distance. As a result, $a_{\mathrm{s} 0}$ is taken as the last observed amplitude shown in Fig. 3(a) for each Mach number, and the values are listed in Table I. Similar to the amplitude, the wavelength of the perturbed shock also increases with increasing the Mach number. However, the rapid increase of wavelength from $M=1.2$ to 1.5 seems to saturate when $M>1.5$. It is worth noting that the wavelength for each Mach number nearly increases linearly with the distance. Thus, it seems reasonable to estimate the wavelength $\lambda_{\mathrm{s} 0}$ by extrapolating the data of the wavelength to the location of the interface, as illustrated in Fig. 3(b). The estimated values of $\lambda_{\mathrm{s} 0}$ for the three Mach numbers, as listed in Table I, agree with those obtained from the Mie scattering pictures of the shocked interfaces, which will be given later, to within $4 \%$.

The amplitude $a_{\mathrm{s}}$ and the wavelength $\lambda_{\mathrm{s}}$ of the perturbed shock from the present experiments are further compared with those from the numerical results by Zhai et al. [16], as shown in Fig. 4. The estimated values of $\lambda_{\mathrm{s} 0}$, which are listed in Table I, are also shown in Fig. 4. Note that the wavelength defined in Ref. [16] is half of that defined in the present study. It is found that there is a good agreement between the experimental and numerical results.

\section{B. Features of the shocked interface}

The evolutions of the shocked interfaces with different initial Atwood numbers at Mach numbers $M=1.2,1.5$, and 1.8 are shown in Figs. 5, 6, and 7, respectively. The initial time, $t=0$, is defined as the time the IS impacts the interface. The initial interface, shown in Fig. 5 by Mie scattering as well as in Fig. 2 by shadowgraphy, is nearly flat with only a very large wavelength curvature. This indicates that the initial interface exhibits desired uniform nature as compared to the perturbed shock. At the early stage, the overall morphologies of the shocked interfaces are similar for all the Atwood numbers and Mach numbers considered. The typical features in the interface morphology are the formations of a $\mathrm{N}_{2}$ cavity and two steps on both sides of the cavity, as indicated by the rectangle and circles in Fig. 6, which result from the nonuniform pressure distribution behind the perturbed shock [14]. As time increases, the depth of the cavity gradually increases while the width of the cavity decreases. In contrast,
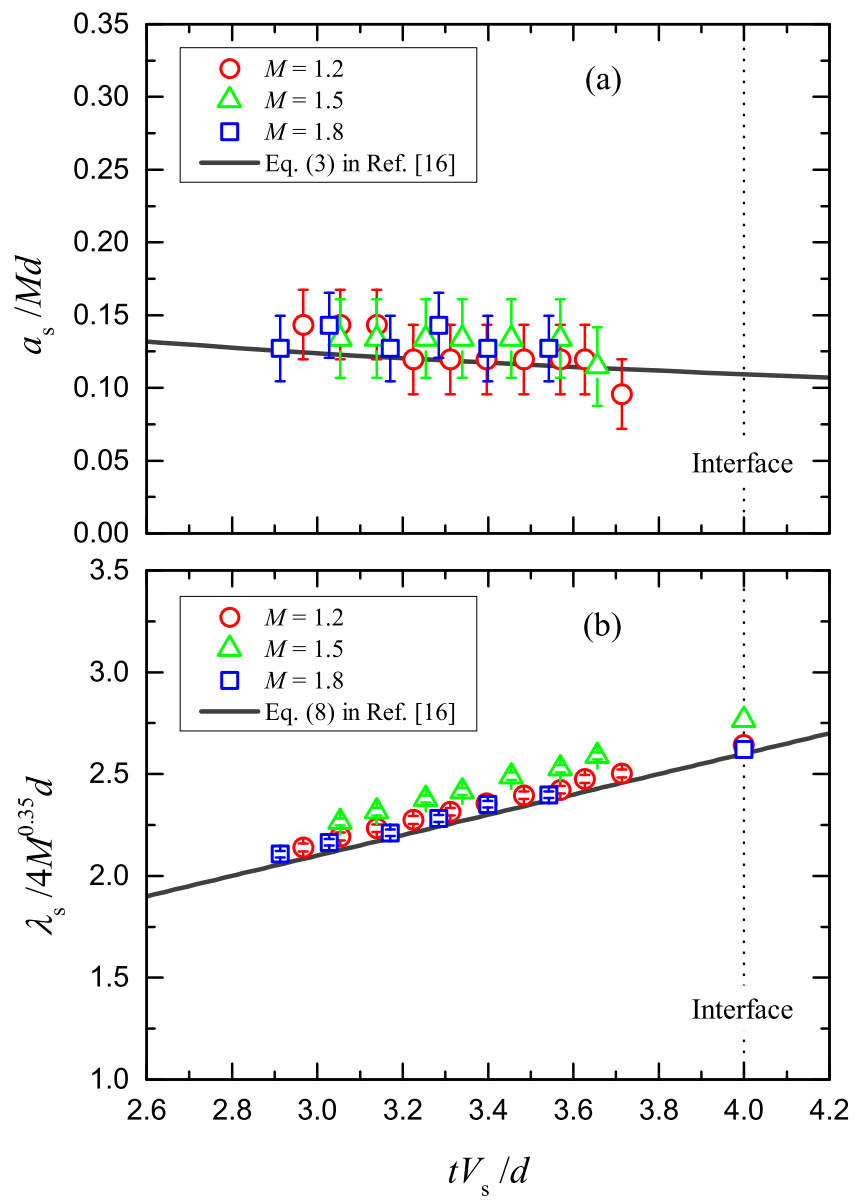

FIG. 4. Comparisons of the amplitude $a_{\mathrm{s}}$ (a) and the wavelength $\lambda_{\mathrm{s}}$ (b) of the perturbed shock from the present experiments with those from the numerical results in Ref. [16]. 
(a) $A=0.22$

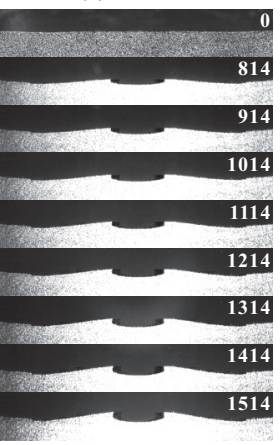

(b) $A=0.50$

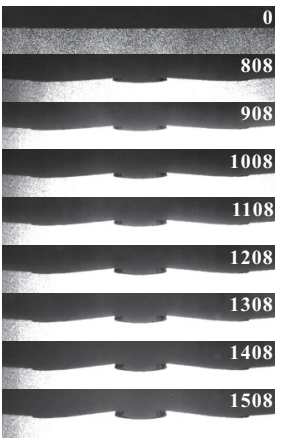

(c) $A=0.68$

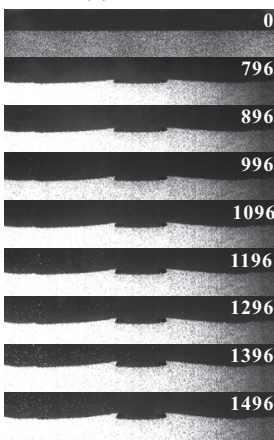

FIG. 5. Mie scattering sequences showing the evolutions of the shocked interfaces with different initial Atwood numbers $A$ at Mach number $M=1.2$. Numbers indicate time in $\mu \mathrm{s}$.

the distance between the two steps changes little with time. It is evident in Figs. 5-7 that, for the same Atwood number, the amplitude of the shocked interface $a_{\mathrm{i}}$, as defined in Fig. 6(b), increases with increasing the Mach number.

Besides the formation of a cavity and two steps, another feature can be observed from Figs. 5-7 that a vortex pair appears to form as both the Atwood number and Mach number increase. An apparent counter-rotating vortex pair emerges on the top of the interface when the Mach number increases to $M=1.8$ and the Atwood number increases to $A=0.68$; see Fig. 7 from $t=283 \mu \mathrm{s}$. This feature may be attributed to the baroclinic vorticity deposition. The vorticity distribution at the early stage of the interaction between a perturbed shock and a uniform interface is shown in Fig. 8. The magnitude of the baroclinic vorticity deposited locally is determined by the non-collinearity of the density gradient $\nabla \rho$ and the pressure gradient $\nabla p$. Note that $\nabla \rho$ across the interface is nearly collinear with $\nabla p$ across the IS and the MS2, therefore very little vorticity is deposited on the two interface segments where the IS and the MS2 pass through. For the MS1, the maximum misalignment between $\nabla \rho$ and $\nabla p$ is at the TP2, though the magnitude of $\nabla p$ decreases continuously along the MS1 to a minimum value at the TP2. Thus, the maximum vorticity is supposed to be deposited at the location where the TP2 impacts the interface, which leads to the formation (a) $A=0.22$

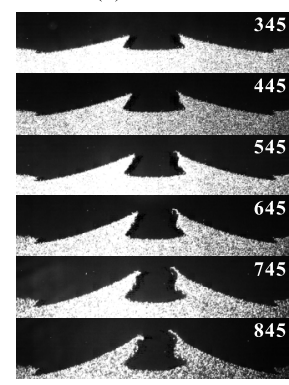

(b) $A=0.50$

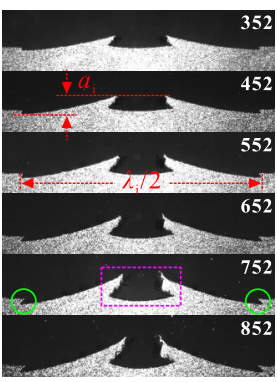

(c) $A=0.68$

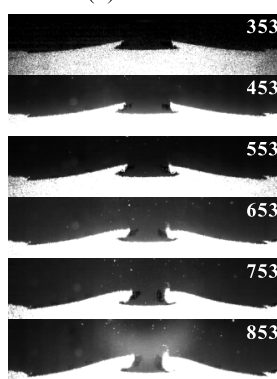

FIG. 6. Mie scattering sequences showing the evolutions of the shocked interfaces with different initial Atwood numbers $A$ at Mach number $M=1.5$. Numbers indicate time in $\mu$ s. Rectangle indicates the $\mathrm{N}_{2}$ cavity and circles indicate the steps. $a_{\mathrm{i}}$ and $\lambda_{\mathrm{i}}$ are the amplitude and wavelength of the shocked interface, respectively. (a) $A=0.22$

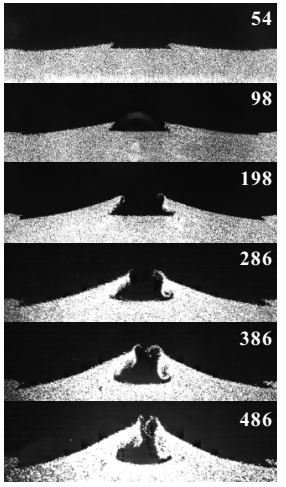

(b) $A=0.50$

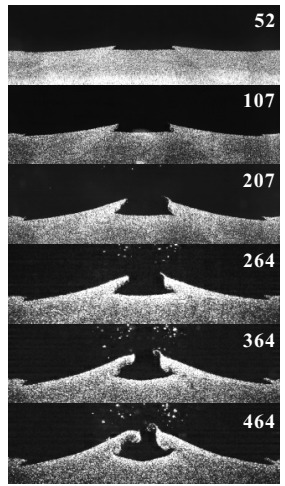

(c) $A=0.68$

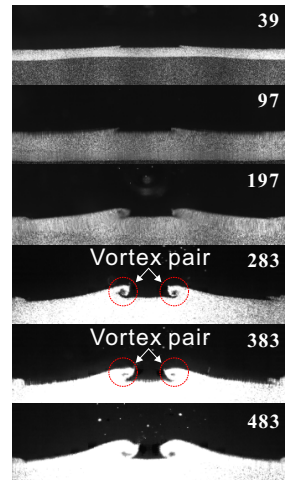

FIG. 7. Mie scattering sequences showing the evolutions of the shocked interfaces with different initial Atwood numbers $A$ at Mach number $M=1.8$. Numbers indicate time in $\mu$ s. Circles indicate the vortex pair.

of a counter-rotating vortex pair near this location. With increasing the Atwood number and Mach number, both the density gradient $\nabla \rho$ and the pressure gradient $\nabla p$ rise. That is the reason why the vortex pair is more remarkable in the case with larger Atwood number and Mach number.

\section{Effect of the Atwood number on the amplitude growth rate}

Variations of the amplitude of the shocked interface $a_{\mathrm{i}}$, indicated in Fig. 6(b), with time $t$ for different initial Atwood numbers and Mach numbers are shown in Fig. 9. The error bars represent the uncertainty of the manual measurement. For the same Mach number, the overall shapes of the amplitude curves are similar for all the Atwood numbers. At low Mach number (e.g., $M=1.2$ ), a clear trend emerges: The amplitudes for all the three Atwood numbers grow linearly with time; see Fig. 9(a). Increase of the Mach number (e.g., $M=1.8$ ) causes an apparent nonlinear effect to occur in the amplitude growth at late times, which results in an obvious decay of the growth rate; see Fig. 9(c). This nonlinear effect can be attributed to the formation of the vortex pair on the top of the shocked interface, as shown in Fig. 7, and is different from that in the classical RM instability resulting

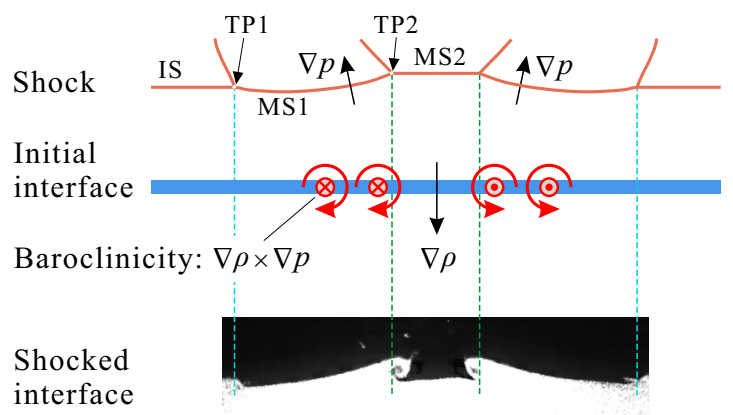

FIG. 8. Schematic of the baroclinic vorticity deposition on the uniform interface. The perturbed shock propagates downward toward the uniform interface and applies a pressure gradient $\nabla p$ across the density gradient $\nabla \rho$, which deposits locally baroclinic vorticity on the interface. 

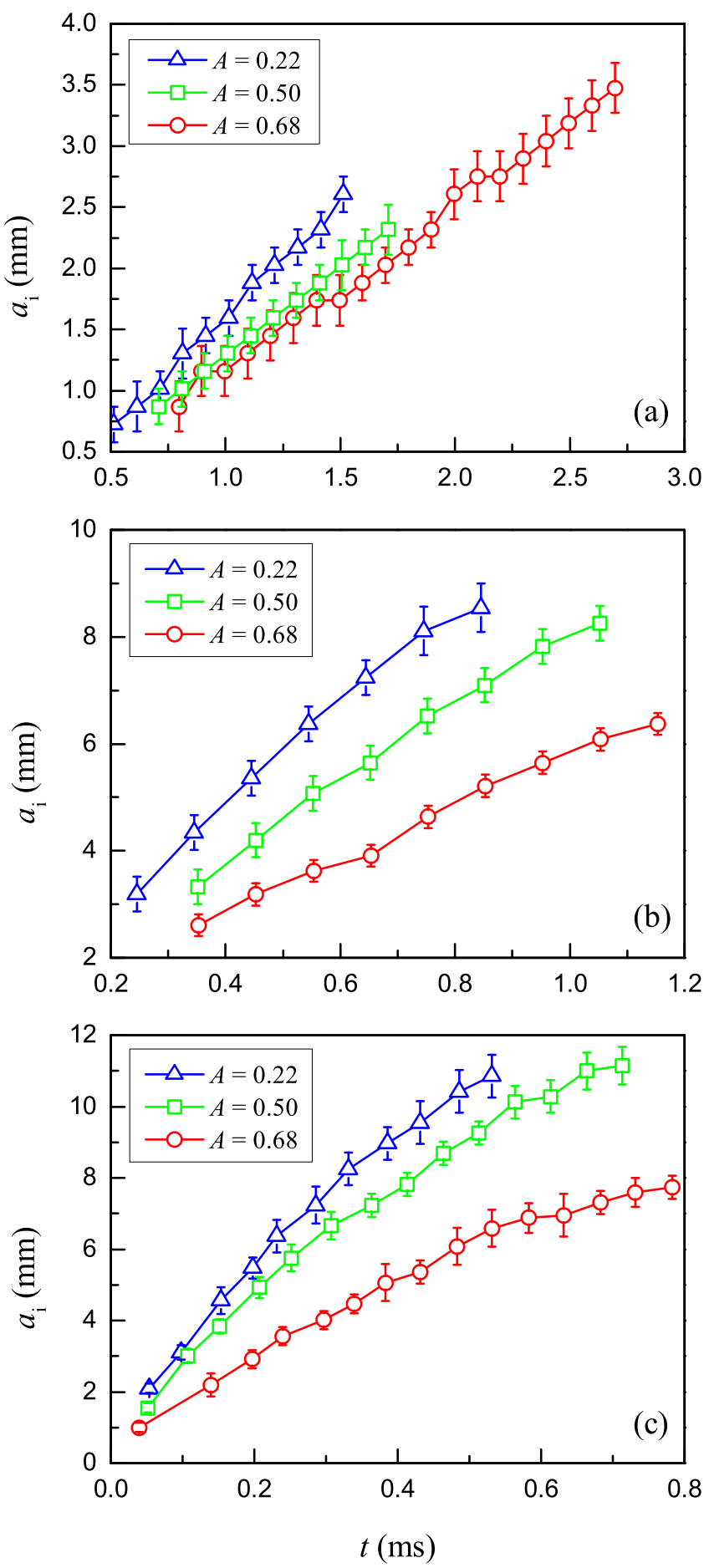

FIG. 9. Variations of the amplitude of the shocked interface $a_{\mathrm{i}}$ with time $t$ for different initial Atwood numbers at Mach number (a) $M=1.2$, (b) $M=1.5$, and (c) $M=1.8$.

in asymmetric spike and bubble development. The larger the Mach number is, the earlier is the formation of the vortex pair, and the earlier the nonlinear effect occurs.

It is interesting to note in Fig. 9 that, for all the three Mach numbers, the amplitude growth rate of the shocked interface $\dot{a}_{\text {exp }}=d a_{\mathrm{i}} / d t$ decreases with increasing the Atwood number $A$. The values of $\dot{a}_{\text {exp }}$ are calculated by linear fitting before it decreases, and the results are listed in Table I. This notable feature is fundamentally different from that in the classical RM instability, where the perturbation amplitude growth rate increases with increasing the Atwood number, as reported in previous experimental studies by Meshkov [2], Brouillette and Sturtevant [25,26], Jourdan and Houas [20], Motl et al. [21], theoretical studies by Mikaelian [27], Yang et al. [28] as well as numerical studies by Zabusky et al. [29] and Lombardini et al. [30]. It should be noted that these previous studies on the classical RM instability focused on not only discontinuous interfaces [2,20,25,27-30], but also continuous interfaces $[21,25,26]$. Moreover, the membraneless, continuous interface in the present study is formed in the same manner as that in Ref. [21].

In previous studies, both Liang et al. [15] and Zhai et al. [16] reported that their empirical models proposed based on Richtmyer's impulsive model could give reasonably good prediction of the instability growth driven by a perturbed shock. However, only a single Atwood number (i.e., air/ $\mathrm{SF}_{6}$ interface) was considered in their experiments. As a result, it is not clear whether a model based on Richtmyer's impulsive model is applicable to other values of Atwood number. To quantitatively investigate the different Atwood number effects between the present instability and the classical RM instabilities, the amplitude growth rates obtained in the present experiments are compared with the estimated values predicted by Richtmyer's impulsive model [1]. Richtmyer's impulsive model has been proved to be a good prescription for the growth rate of the classical RM instability in the case of small perturbation amplitude and weak to moderately strong shocks [28]. According to the model, the amplitude growth rate is given by

$$
\dot{a}=a_{0} k A^{+} \Delta V,
$$

where $a_{0}$ is the initial perturbation amplitude, $k=2 \pi / \lambda_{0}$ is the wave number of the perturbation with initial wavelength $\lambda_{0}, A^{+}$is the post-shock Atwood number and $\Delta V$ is the velocity jump of the shocked interface. First, the amplitude growth rate is calculated similarly as in our previous work [14], where the pre-interface amplitude of the perturbed shock is assumed to be equivalent to the pre-shock amplitude of the perturbed interface. Accordingly, the amplitude $a_{\mathrm{s} 0}$ and the wavelength $\lambda_{\mathrm{s} 0}$ of the perturbed shock are considered as the initial amplitude $a_{0}$ and wavelength $\lambda_{0}$ of the perturbed interface, respectively. Note that $a_{\mathrm{s} 0}$ is significantly smaller than $\lambda_{\mathrm{s} 0}\left(a_{\mathrm{s} 0} / \lambda_{\mathrm{s} 0} \ll 1\right)$ for all the three Mach numbers, which is expected to satisfy the assumption of small perturbation amplitude. Thus, the amplitude growth rate can be calculated by substituting $a_{\mathrm{s} 0}$ and $\lambda_{\mathrm{s} 0}$ for $a_{0}$ and $\lambda_{0}$ in Eq. (1), respectively, and the values, designated as $\dot{a}_{\text {eq } 1}$, are shown in Table I. It is clear that the dependence of $\dot{a}_{\text {eq1 }}$ on the Atwood number $A$ predicted by the theoretical model is significantly different from its experimental counterpart $\dot{a}_{\text {exp }}$. For $A=0.68$ (i.e., $\mathrm{N}_{2} / \mathrm{SF}_{6}$ interface), $\dot{a}_{\text {eq } 1}$ is larger than $\dot{a}_{\text {exp }}$, which is consistent with the findings of Zou et al. [14] and Liang et al. [15]. For continuous interface, experimental results by Brouillette and Sturtevant [26] demonstrated that the growth rate is reduced by the presence of a finite density gradient on the interface. Moreover, the larger the diffusion thickness is, the smaller the growth rate is. Similar behavior was also experimentally observed by Motl et al. [21], and they concluded that a growth 
reduction factor should be introduced to correct the impulsive model for the case of a continuous interface, since the impulsive model is based on the discontinuous interface. The diffusion effect inherent to the continuous interface may partly explain the result that $\dot{a}_{\text {eq1 }}$ is larger than $\dot{a}_{\text {exp }}$ for $A=0.68$; however, it cannot explain why $\dot{a}_{\text {eq } 1}$ is smaller than $\dot{a}_{\text {exp }}$ for $A=0.22$ (i.e., $\mathrm{N}_{2} / \mathrm{CO}_{2}$ interface), especially at higher Mach numbers.

The initial compression of the interface and of the fluids should be taken into account to predict the amplitude growth rate, as suggested by Richtmyer [1]. In this work, we also attempt to predict the growth rate by equating the amplitude of the shocked interface after the passage of the perturbed shock to the post-shock amplitude of the perturbed interface in the classical RM instability. The post-shock initial amplitude $a_{\mathrm{i} 0}$ can be estimated based on the one-dimensional gas dynamics. As illustrated in Fig. 2(a), $a_{\mathrm{s} 0}$ is the amplitude of the perturbed shock corresponding to the time when the incident shock (IS) impacts the interface. The time taken for the perturbed shock to completely pass through the interface is $a_{\mathrm{s} 0} / V_{\mathrm{TP} 2}$, where $V_{\mathrm{TP} 2}$ is the streamwise velocity of the triple point TP2 and its value is measured from the shadowgraph pictures. During this time interval, the interface impacted by the IS moves a distance of $a_{\mathrm{i} 0}$ with velocity of $\Delta V$. Thus, we have $a_{\mathrm{i} 0} / \Delta V=$ $a_{\mathrm{S} 0} / V_{\mathrm{TP} 2}$, which can be rewritten as

$$
a_{\mathrm{i} 0}=a_{\mathrm{s} 0} \frac{\Delta V}{V_{\mathrm{TP} 2}} .
$$

The estimated values of $a_{\mathrm{i} 0}$ are listed in Table I. The postshock initial wavelength of the interface $\lambda_{\mathrm{i} 0}$ is measured when the two interface steps can be clearly resolved in the Mie scattering pictures, and the values are also listed in Table I. Since there is only a slight temporal variation in the wavelength of the shocked interface $\lambda_{\mathrm{i}}$, as compared with the variation in the amplitude $a_{\mathrm{i}}, \lambda_{\mathrm{i} 0}$ is considered as the initial wavelength of the perturbed interface $\lambda_{0}$. Obviously, from Eq. (2) and Table I, $a_{\mathrm{i} 0}$ is smaller than $a_{\mathrm{s} 0}$ and $\lambda_{\mathrm{i} 0}$ is slightly larger than $\lambda_{\mathrm{s} 0}$, therefore $a_{\mathrm{i} 0}$ also satisfies the assumption of small perturbation amplitude. Thus, the amplitude growth rate can be calculated by substituting $a_{\mathrm{i} 0}$ and $\lambda_{\mathrm{i} 0}$ for $a_{0}$ and $\lambda_{0}$ in Eq. (1), respectively, and the values, designated as $\dot{a}_{\text {eq } 2}$, are also shown in Table I. It is found that $\dot{a}_{\text {eq2 }}$ is much smaller than $\dot{a}_{\text {exp }}$. Moreover, the dependence of $\dot{a}_{\text {eq2 } 2}$ on $A$ predicted by the theoretical model is similar to that of $\dot{a}_{\text {eq } 1}$ on $A$, and is still different from the experimental counterpart. Consequently, the present experimental results provide quantitative evidence of the significantly different characteristics between the interface instability driven by a perturbed shock and the classical RM instability, in terms of the dependence of the amplitude growth rate on the Atwood number. Furthermore, it is believed that the diffusion effect inherent to the continuous interface is not responsible for these different characteristics.

\section{Physics underlying the instability}

When the perturbed shock impacts the uniform interface, it impulsively imprints perturbation on the interface. The interaction of the perturbed shock with the uniform interface can be approximated to numerous oblique shock segments with infinitesimal length hitting the uniform interface. Consider

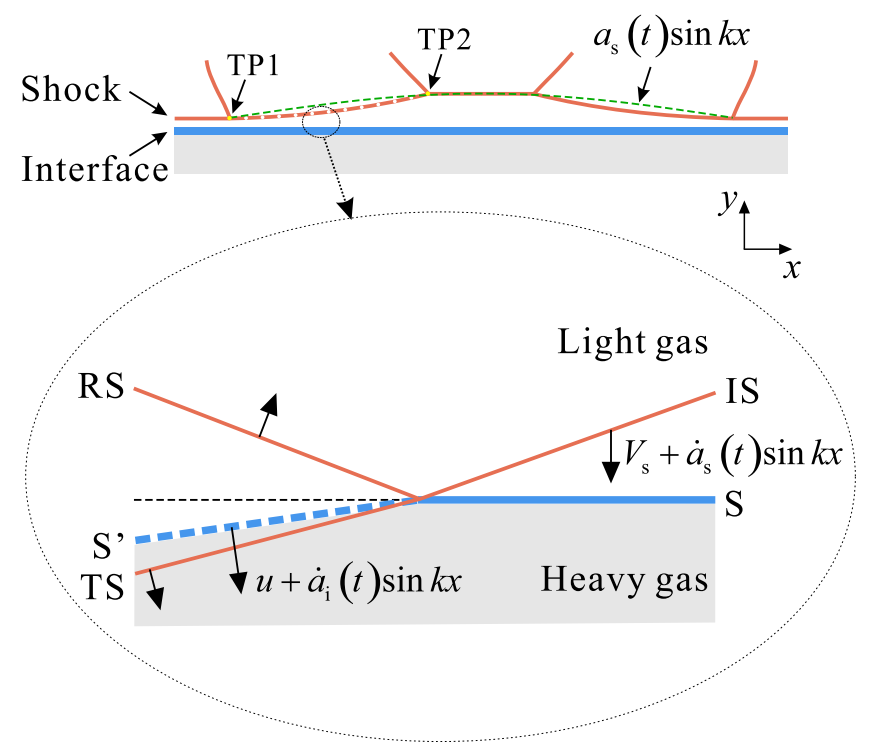

FIG. 10. Schematic of the transmission and reflection of an oblique shock segment crossing a uniform interface initially at rest. $\dot{a}_{\mathrm{s}}(t)$ and $\dot{a}_{\mathrm{i}}(t)$ are the amplitude growth rates of the rippled shock and the shocked interface, respectively. $V_{\mathrm{s}}$ and $u$ are the velocities of the shock and the shocked interface, respectively. Notation: IS, incident shock; RS, reflected shock; TS, transmitted shock; S, initial interface; $S^{\prime}$, shocked interface.

the transmission and reflection of an oblique shock segment crossing a uniform interface initially at rest, as illustrated in Fig. 10. If we approximate the perturbation of the perturbed shock as $a_{\mathrm{s}}(t) \sin k x$, then the initial amplitude growth rate of an interface $\dot{a}_{\mathrm{i}}\left(t_{0}\right)$ imprinted by the perturbed shock, taking into account only the first-order terms, is given by Ishizaki et al. [12] as

$$
\dot{a}_{\mathrm{i}}\left(t_{0}\right)=\frac{u}{V_{\mathrm{s}}} \dot{a}_{\mathrm{s}}\left(t_{0}\right),
$$

where $u$ and $V_{\mathrm{s}}$ are the velocities of the shocked interface and the perturbed shock, respectively, $\dot{a}_{\mathrm{s}}(t)$ is the amplitude growth rate of the perturbed shock and $t_{0}$ corresponds to the time when the shock impacts the interface. Equation (3) implies that, for the same incident perturbed shock (i.e., the same $V_{\mathrm{s}}$ and $\left.\dot{a}_{\mathrm{s}}\left(t_{0}\right)\right)$, the amplitude growth rate of the shocked interface is directly proportional to the velocity of the shocked interface, i.e., $\dot{a}_{\mathrm{i}} \propto u$. Note that $u$ is inversely proportional to the Atwood number $A$ considered in the present experiments as indicated in Table I, i.e., $u \propto 1 / A$. As a result, the amplitude growth rate of the shocked interface goes as $\dot{a}_{\mathrm{i}} \propto 1 / A$. This qualitatively explains our experimental results that the amplitude growth rate of the shocked interface decreases with increasing the Atwood number.

Equation (3) also implies that the amplitude growth of the shocked interface only depends on the velocity of the shocked interface $u$ for the same perturbed shock. Therefore, it may be appropriate to scale the time by $u$. Here, the velocity jump of the shocked interface $\Delta V$ induced by the incident shock IS is used instead of $u$, considering the complex wave patterns of the perturbed shock. Variations of the normalized amplitude of the shocked interface, $a_{\mathrm{i}} / a_{\mathrm{s} 0}$, with the normalized time, 

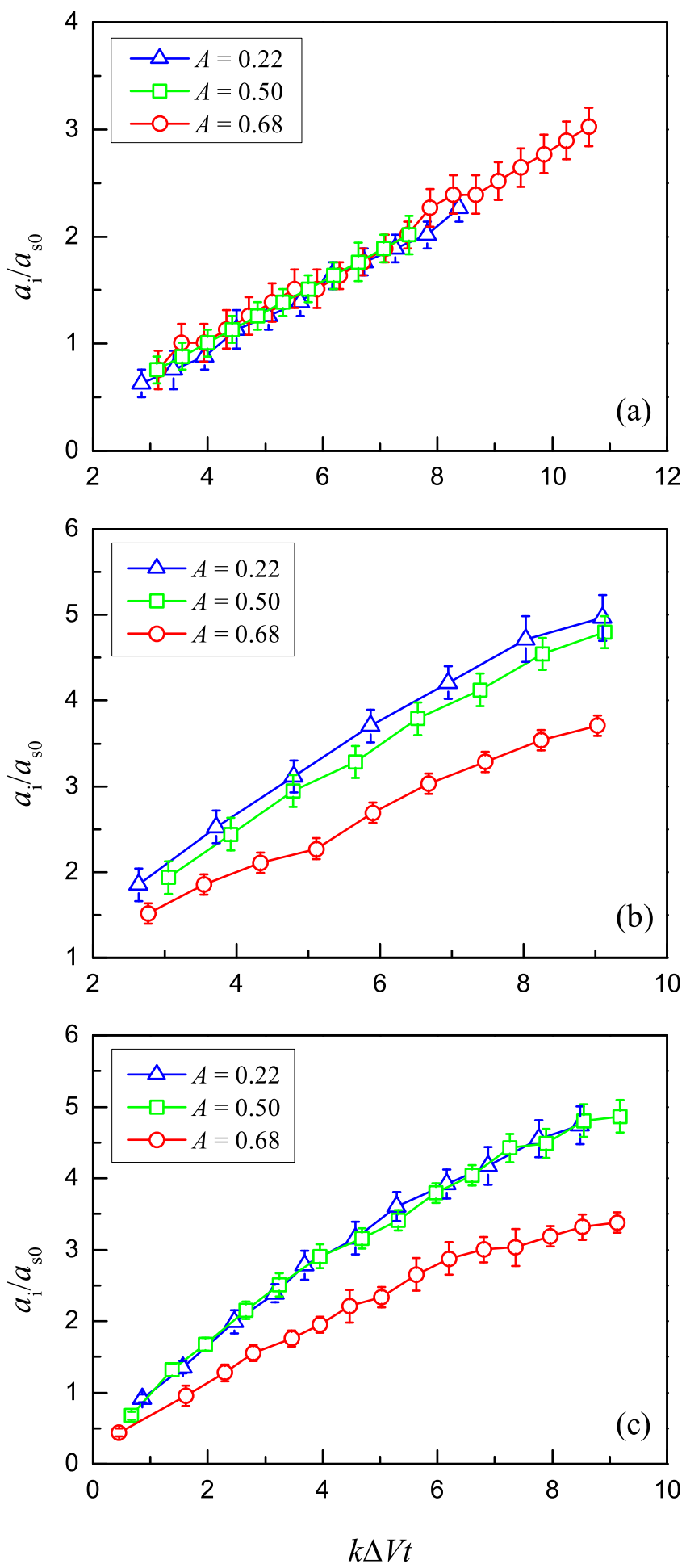

FIG. 11. Variations of the normalized amplitude of the shocked interface, $a_{\mathrm{i}} / a_{\mathrm{s} 0}$, with the normalized time, $k \Delta V t$, for different initial Atwood numbers at Mach number (a) $M=1.2$, (b) $M=1.5$, and (c) $M=1.8 . k=2 \pi / \lambda_{\mathrm{s} 0}$ is the wave number.

$k \Delta V t$, are shown in Fig. 11 , where $k=2 \pi / \lambda_{\mathrm{s} 0}$. As expected, all the experimental results collapse onto a single straight line at Mach number $M=1.2$, irrespective of the initial Atwood number difference. We also found that the collapse cannot be achieved if the natural time scale commonly used for the classical RM instability, $1 /\left(k A^{+} \Delta V\right)[17,31,32]$, was used to scale the time. As $M$ increases, the collapse of all the data fails. The amplitudes for Atwood numbers $A=0.22$ and $A=0.50$ still fall closely onto a single curve, but leaving an apparent discrepancy with the data of $A=0.68$. It seems that $\Delta V$ is a suitable time scaling only for the weak shock (i.e., $M=1.2$ ). Note that Eq. (3) only describes the regime that the perturbed shock impulsively imprints perturbation on the interface, but does not take into account the effects of the continuous pressure perturbations after the passage of the perturbed shock on the evolution of the shocked interface. In fact, the latter plays an important role in both the classical RM instability [1] and the instability driven by a nonuniform shock [12]. As pointed out by Richtmyer [1] and Fraley [33], the effect of compressibility cannot be neglected anymore for relativley strong shocks. Moreover, the nonlinear decay in the amplitude growth rate caused by the baroclinic vorticity deposition, especially in higher Mach number cases, is also not accounted for by Eq. (3). These may be the reasons why the experimental data fails to collapse in higher Mach number cases.

It is evident from the foregoing discussion that the physics underlying the present instability driven by a perturbed shock is different from that underlying the classical RM instability caused by a planar shock. In the classical RM instability, the sources driving the growth of the perturbation on the interface are the pressure perturbations behind the two overstable shocks, i.e., the transmitted shock and the reflected shock, as pointed out by Richtmyer [1] and Fraley [33]. However, in the present instability, the velocity perturbation is first impulsivley imprinted on the interface by the perturbed shock at the impinging moment, and then the growth of the interface perturbation is driven by the pressure perturbations behind the transmitted and reflected shocks. The different underlying physics results in different behaviors of the perturbation growth. One of the major differences is the initial amplitude growth rate of the shocked interface $\dot{a}_{\mathrm{i}}\left(t_{0}\right)$. In the classical RM instability, as pointed out by Richtmyer [1], $\dot{a}_{\mathrm{i}}\left(t_{0}\right)$ is zero because the forward velocity imparted by the planar shock to the crest of the corrugation on the interface is the same as the forward velocity imparted to the trough. However, in the present instability, $\dot{a}_{\mathrm{i}}\left(t_{0}\right)$ depends on the amplitude growth rate of the perturbed shock $\dot{a}_{\mathrm{s}}\left(t_{0}\right)$ at the impinging moment, as indicated by Eq. (3), and thus it is usually not zero. Another major difference lies in the asymptotic value of $\dot{a}_{\mathrm{i}}$. In the classical RM instability, $\dot{a}_{\text {i }}$ starts from zero and approaches an nonzero asymptotic value at large times $[1,33]$. However, in the present instability, asymptotic value of $\dot{a}_{\text {i }}$ may be zero, even if perturbations do exist on the perturbed shock front at the impinging moment [12]. This means that the shocked interface is stable, which will never occur in the classical RM instability.

\section{CONCLUSIONS}

The membraneless uniform interface subjected to a perturbed shock wave is experimentally investigated over a range of Atwood numbers $0.22 \leqslant A \leqslant 0.68$ and Mach numbers $1.2 \leqslant M \leqslant 1.8$ using a vertical shock tube. The perturbed shock is generated by the diffraction of a planar incident shock 
over a rigid cylinder. The wave patterns of the perturbed shock are captured by high-speed shadowgraphy while the evolution of the shocked interface is quantitatively characterized by planar Mie scattering.

Besides the general formations of a cavity and two steps, an apparent counter-rotating vortex pair emerges on the top of the shocked interface due to the baroclinic vorticity deposition, as both the Atwood number and Mach number increase. An interesting observation shows that the amplitude growth rate of the shocked interface decreases with increasing the Atwood number for all the three Mach numbers. This notable feature is fundamentally different from the Atwood number effects in the classical RM instability. Two kinds of amplitude growth rates are calculated by Richtmyer's impulsive model, but both exhibit apparent disagreement with the experimental results. The Atwood number effect on the amplitude growth rate of the shocked interface can be qualitatively interpreted by the approximate solution of an oblique shock hitting a uniform interface. Using the velocity jump of the shocked interface as a time scaling, all the experimental amplitude data collapse onto a single straight line at Mach number $M=1.2$. The failure of the collapse of data in higher Mach number cases is attributed to the effects of the pressure perturbations as well as the baroclinic vorticity deposition. The present experimental results provide a better understanding of the physics underlying the interaction between a shock wave with complex shock stucture and gas inhomogeneities, and are helpful for code validations.

\section{ACKNOWLEDGMENTS}

This work was supported by the Science Challenge Project (Grant No. TZ2016001) and the National Natural Science Foundation of China (Projects No. 11472253, No. 11602247 , No. 11772309, and No. 11672277).
[1] R. D. Richtmyer, Commun. Pure Appl. Math. 13, 297 (1960).

[2] E. E. Meshkov, Fluid Dyn. 4, 101 (1969).

[3] L. Smarr, J. R. Wilson, R. T. Barton, and R. L. Bowers, Astrophys. J. 246, 515 (1981).

[4] V. Rana, H. Lim, J. Melvin, J. Glimm, B. Cheng, and D. H. Sharp, Phys. Rev. E 95, 013203 (2017).

[5] N. J. Zabusky, Annu. Rev. Fluid. Mech. 31, 495 (1999).

[6] M. Brouillette, Annu. Rev. Fluid. Mech. 34, 445 (2002).

[7] D. Ranjan, J. Oakley, and R. Bonazza, Annu. Rev. Fluid. Mech. 43, 117 (2011).

[8] Y. Zhou, Phys. Rep. 720-722, 1 (2017).

[9] Y. Zhou, Phys. Rep. 723-725, 1 (2017).

[10] Z. Zhai, L. Zou, Q. Wu, and X. Luo, Proc. IMechE Part C: J. Mech. Eng. Sci. 232, 2830 (2018).

[11] J. O. Kane, H. F. Robey, B. A. Remington, R. P. Drake, J. Knauer, D. D. Ryutov, H. Louis, R. Teyssier, O. Hurricane, D. Arnett, R. Rosner, and A. Calder, Phys. Rev. E 63, 055401 (2001).

[12] R. Ishizaki, K. Nishihara, H. Sakagami, and Y. Ueshima, Phys. Rev. E 53, R5592 (1996).

[13] W. Zhang, Q. Wu, L. Zou, X. Zheng, X. Li, X. Luo, and J. Ding, Phys. Rev. E 98, 043105 (2018).

[14] L. Zou, J. Liu, S. Liao, X. Zheng, Z. Zhai, and X. Luo, Phys. Rev. E 95, 013107 (2017).

[15] Y. Liang, J. Ding, Z. Zhai, T. Si, and X. Luo, Phys. Fluids 29, 086101 (2017).

[16] Z. Zhai, Y. Liang, L. Liu, J. Ding, X. Luo, and L. Zou, Phys. Fluids 30, 046104 (2018).
[17] Y. Zhou, W. H. Cabot, and B. Thornber, Phys. Plasmas 23, 052712 (2016).

[18] M. Jones and J. Jacobs, Phys. Fluids 9, 3078 (1997).

[19] P. Puranik, J. Oakley, M. Anderson, and R. Bonazza, Shock Waves 13, 413 (2004).

[20] G. Jourdan and L. Houas, Phys. Rev. Lett. 95, 204502 (2005).

[21] B. Motl, J. Oakley, D. Ranjan, C. Weber, M. Anderson, and R. Bonazza, Phys. Fluids 21, 126102 (2009).

[22] A. E. Bryson and R. W. F. Gross, J. Fluid Mech. 10, 1 (1961).

[23] W. H. Heilig, Phys. Fluids 12, 154 (1969).

[24] K. Luo, Y. Luo, T. Jin, and J. Fan, Sci. China Tech. Sci. 60, 1423 (2017).

[25] M. Brouillette and B. Sturtevant, Physica D (Amsterdam) 37, 248 (1989).

[26] M. Brouillette and B. Sturtevant, J. Fluid Mech. 263, 271 (1994).

[27] K. O. Mikaelian, Physica D (Amsterdam) 36, 343 (1989).

[28] Y. Yang, Q. Zhang, and D. H. Sharp, Phys. Fluids 6, 1856 (1994).

[29] N. J. Zabusky, A. D. Kotelnikov, Y. Gulak, and G. Peng, J. Fluid Mech. 475, 147 (2003).

[30] M. Lombardini, D. J. Hill, D. I. Pullin, and D. I. Meiron, J. Fluid Mech. 670, 439 (2011).

[31] B. Thornber and Y. Zhou, Phys. Rev. E 86, 056302 (2012).

[32] M. Lombardini, D. I. Pullin, and D. I. Meiron, J. Fluid Mech. 690, 203 (2012).

[33] G. Fraley, Phys. Fluids 29, 376 (1986). 